\title{
Integrated Echocardiographic Imaging of Giant Atrial Myxoma
}

Roberto Cemin ${ }^{1 *}$, Renato Di Gaetano', Giovanni Battista Luciani ${ }^{2}$ and Andrea Erlicher ${ }^{1}$

${ }^{1}$ Department of Cardiology, Bolzano General Hospital, Bolzano, Italy

${ }^{2}$ Division of Cardiac Surgery, University of Verona, Verona, Italy

Keywords: Cardiac tumor; 3D echocardiography; Contrast echocardiography

\section{Description}

A 50-year-old woman with a history of pituitary adenoma underwent transthoracic echocardiography (TTE) to assess cardiac valve function after previous treatment with cabergoline. TTE ruled out valve pathology, but showed a large rounded mass within the left atrium (Figure 1). The mass adhered to the interatrial septum and projected toward the mitral valve during ventricular diastole without generating flow obstruction. The findings were suggestive of left atrial myxoma. In order to evaluate vascularisation of the mass, contrast echocardiography was performed using a second-generation contrast agent (SonoVue ${ }^{\oplus}$ Bracco Imaging, Milan, Italy) with real time perfusion imaging. The slow contrast refilling of the mass after a high-energy ultrasound pulse was suggestive of low vascularisation (Figure 1), typical of benign tumors [1].

Transesophageal chocardiography (TEE) was performed to further characterize morphology, volume and site of adhesion of the mass and

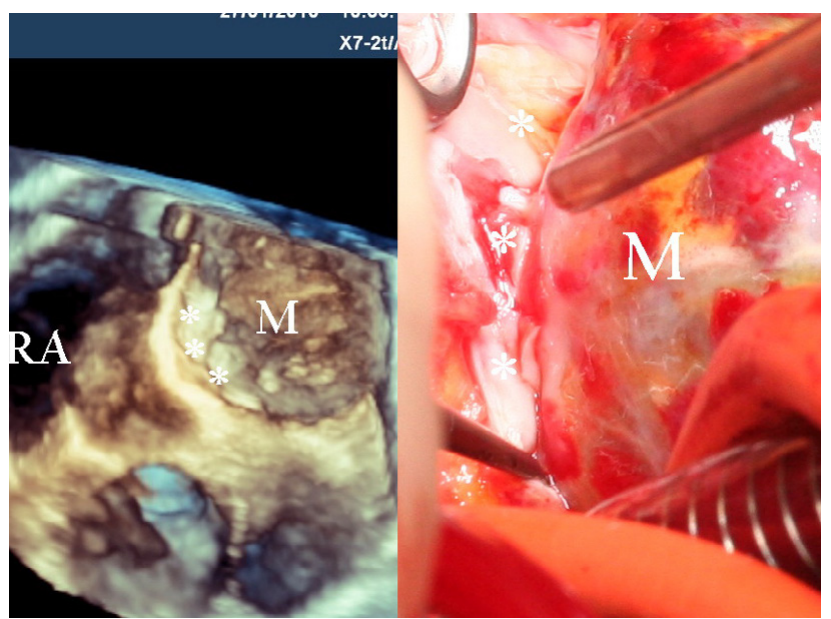

Figure 1: The slow contrast refilling of the mass after a high-energy ultrasound pulse was suggestive of low vascularization. to aide planning [2]. 2D TEE confirmed diagnosis of giant left atrial mass and attachment to the atrial septum. The real-time 3D (RT-3D) TEE allowed to display a virtual atriotomic view, familiar and valuable to the surgeon. Particularly useful was the assessment of the very broad adhesion of the mass, which was unusually located on the atrial septum and roof of the left atrium (Figure 2). Radical excision was performed using a biatrial approach. Firstly, via right atriotomy, the septum primum was incised circumferentially to mobilize the septal adhesion of the mass. Secondly, via left atriotomy, the insertion onto the roof of the left atrium was excised along with a patch of left atrial free wall. Histology was compatible with myxoma. The wall defect was repaired primarily, while the atrial septal defect was patched with autologous pericardium.

\section{References}

1. Kirkpatrick JN, Wong T, Bednarz JE, Spencer KT, Sugeng L, et al. (2004) Differential diagnosis of cardiac masses using contrast echocardiographic perfusion imaging. J Am Coll Cardiol 43: 1412-1419.

2. Scohy TV, Lecomte PV, Mc Ghie J (2008) Intraoperative real time threedimensional transesophageal echocardiographic evaluation of right atrial tumor. Echocardiography 25: 646-649.

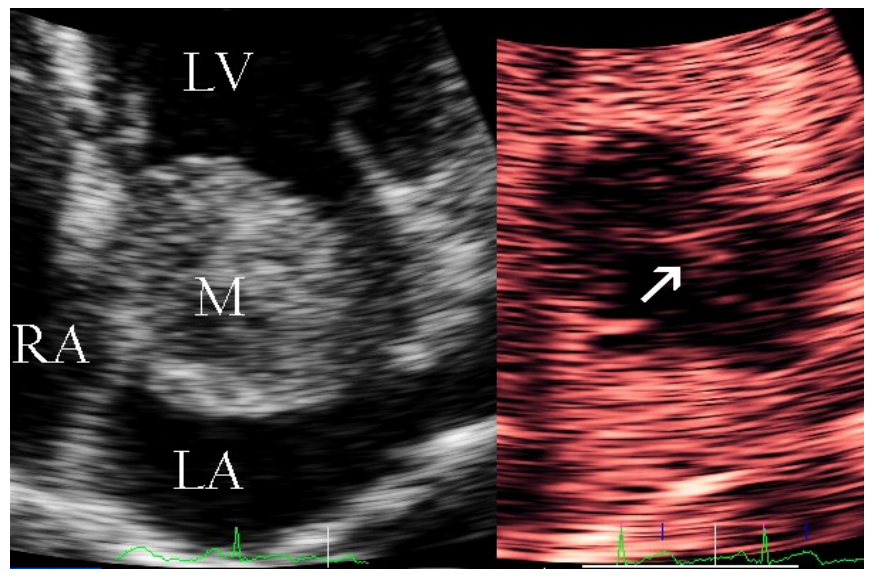

Figure 2: The real-time 3D (RT-3D) TEE allowed to display a virtual atriotomic view, familiar and valuable to the surgeon. Particularly useful was the assessment of the very broad adhesion of the mass, which was unusually located on the atrial septum and roof of the left atrium.

${ }^{*}$ Corresponding author: Roberto Cemin, Department of Cardiology, Bolzano General Hospital, Bolzano, Italy, Tel: +390471909985; Fax: +390471909997; E-mail: roberto.cemin@asbz.it

Received November 22, 2016; Accepted January 15, 2017; Published January 17,2017

Citation: Cemin R, Gaetano RD, Luciani GB, Erlicher A (2017) Integrated Echocardiographic Imaging of Giant Atrial Myxoma. J Cardiovasc Dis Diagn 5: i104. doi: 10.4172/2329-9517.1000i104

Copyright: $\odot 2017$ Cemin R, et al. This is an open-access article distributed under the terms of the Creative Commons Attribution License, which permits unrestricted use, distribution, and reproduction in any medium, provided the original author and source are credited. 